\title{
THE SIMILARITY BOUNDARY OF A SELF-SIMILAR SET
}

\author{
J.E. KEESLING AND C. KRISHNAMURTHI
}

\begin{abstract}
We define the similarity boundary of a self-similar set and use it to analyze the properties of self-similar sets in the general setting of any complete metric space. The similarity boundary is an attempt at extending the concept of the topological boundary in a way that is consistent with our intuitive understanding of the term boundary. We also show how with the analysis of the similarity boundary, we can restrict ourselves to the space $K$ and disregard the ambient space.
\end{abstract}

\section{§1. INTRODUCTION}

We analyze self-similar sets which arise as the invariant sets or attractors of a finite collection of similitudes on a complete metric space. Let $\left\{f_{1}, \cdots, f_{N}\right\}$ be a collection of contracting similitudes with contraction factors $\left\{r_{1}, \cdots, r_{N}\right\}$, defined on a complete metric space $(X, d)$. Then there exists a unique, non-empty, compact subset $K \subseteq X$ such that $K=\bigcup_{i=1}^{N} f_{i}(K)$. This set $K$, which is the attractor of the collection of similitudes is called the self-similar set or the invariant set of the similitudes. The collection of similitudes is called an iterated function system (IFS).

There are a number of dimensions associated with a self-similar set. Of these, one of the most important and widely studied dimensions is the Hausdorff dimension and the associated Hausdorff measure. In fact, one of the earliest characterizations of self-similar sets with positive Hausdorff measure gave rise to the famous open set condition (OSC) set down by Hutchinson[8]. If there exists a non-empty, bounded, open set $G$ in $\mathbb{R}^{n}$ such that $f_{i}(G) \subset G$ and $f_{i}(G) \cap f_{j}(G)=\emptyset$ for all $i \neq j$ then the set of similitudes $\left\{f_{1}, \cdots, f_{N}\right\}$ is said to satisfy the OSC. In the setting of the Euclidean metric space, the OSC is equivalent to finite positive Hausdorff measure in dimension $\alpha$ where $\alpha$ is the similarity dimension (to be defined later). Since positive Hausdorff measure implied that the Hausdorff dimension coincided with the similarity dimension, the OSC guaranteed that the Hausdorff dimension was $\alpha$. But in the general setting of any complete metric space, the OSC does not even imply that the Hausdorff dimension is $\alpha$. This led to a strengthening of the OSC - called the strong open set condition (SOSC) - attributed to Lalley. In addition to the OSC being satisfied, the SOSC requires that the open set $G$ be such that $G \cap K \neq \emptyset$. The SOSC

1991 Mathematics Subject Classification. Primary 28A78, 54E40; Secondary 54H15.

Key words and phrases. Hausdorff dimension, iterated function systems, $\beta$-space, self-similar fractals, image measure, sequence space, sef-similar tiles..

Typeset by $\mathcal{A} \mathcal{M S}-\mathrm{T}_{\mathrm{E}} \mathrm{X}$ 
implies that the Hausdorff dimension is $\alpha$ but does not imply that the Hausdorff measure is positive. Subsequently, there have been other characterizations of self-similar sets with positive Hausdorff measures both in the setting of Euclidean space and any complete metric space in general $([1],[15])$.

Both the OSC and the SOSC are weaker versions of the requirement that: $f_{i}(K) \cap f_{j}(K)=$ $\emptyset$ for all $i \neq j$. When this last condition holds $\operatorname{dim}_{H} K=\alpha$ and the Hausdorff $\alpha$-measure

of $K$ is finite and positive whatever the ambient space. One can think of the OSC and SOSC as attempts to guarantee that $\operatorname{dim}_{H} K=\alpha$ while allowing $f_{i}(K) \cap f_{j}(K)$ to be possibly nonempty for $i \neq j$. This has led to serious interest in intersections of the form $f_{i}(K) \cap f_{j}(K)$ in a quest to further weaken the OSC and SOSC. In this paper we study these intersections through a measure on $K$ called the image measure (to be defined in section 2 ). We define what we call the similarity boundary of $K$ which is an attempt at extending the concept of a topological boundary. This approach to self-similar sets is useful because we have been able to restrict ourselves to the set $K$. In other words, the ambient space $X$ does not play a role. This is significant because, in the context of $K$, the open set in the SOSC is required to be open only in $K$. Moreover, we prove subsequently that under certain conditions on the similarity boundary, the SOSC (restricted to $K$ ) is indeed satisfied. In order to highlight the usefulness of the similarity boundary in analyzing the properties of self-similar sets, we conclude with a few examples, chief among them the Hilbert space example found in [15]. Also, as we show later, in the case of self-similar tiles, the similarity boundary that we define, under certain conditions, does indeed coincide with the topological boundary.

\section{$\S 2$. Definitions and Notation}

Let $(X, d)$ be a complete metric space and let $f_{i}: X \longrightarrow X, i=1, \cdots, N$ be contracting similitudes with contraction factors $0<r_{i}<1$. Let $K \subset X$ denote the the unique nonempty, compact subset of $X$ such that $K=\bigcup_{i=1}^{N} f_{i}(K) . K$ is called the self-similar set or the invariant set of the IFS. We denote by $D$ the Hausdorff metric on the space of non-empty, compact subsets of $X$. The Hausdorff metric is defined the following way:

$$
D(F, K)=\inf \{\epsilon: U(F, \epsilon) \supset K \text { and } U(K, \epsilon) \supset F\}
$$

where $U(A, \epsilon)$ denotes the $\epsilon$-neighbourhood of $A$.

We define $\mathcal{I}_{n}=\left\{I=\left(i_{1}, \ldots, i_{n}\right):|I|=n\right\}$ for $n \geq 0$ where the length of $I$ is denoted by $|I|$ and $i_{k} \in\{1, \ldots, N\}$. For $n=0$ the empty string $\emptyset$ is assumed to be an element of the set. We let $\mathcal{I}=\bigcup_{n=0}^{\infty} \mathcal{I}_{n}$.

We denote the concatenation of two strings $I=\left(i_{1}, \cdots, i_{n}\right)$ and $J=\left(j_{1}, \cdots, j_{m}\right)$ the following way: $I J=\left(i_{1}, \cdots, i_{n}, j_{1}, \cdots, j_{m}\right)$.

$I$ and $J$ are termed incomparable if there exists no $L$ such that $I=J L$ or $J=I L$. We denote incomparable strings the following way: $I \Varangle J$. The following is a list of short-hand notation that will be used in the paper:

$$
\begin{aligned}
f_{I}(K) & =f_{i_{1}} \circ \cdots \circ f_{i_{n}}(K) \\
K_{I} & =f_{I}(K)
\end{aligned}
$$




$$
\begin{gathered}
r_{I}= \begin{cases}r_{i_{1}} \cdots r_{i_{n}} & \text { for } n>0 \\
1 & \text { for } n=0\end{cases} \\
r_{I}^{*}= \begin{cases}r_{i_{1}} \cdots r_{i_{n-1}} & \text { for } n>1 \\
1 & \text { for } n=1 \\
\infty & \text { for } n=0\end{cases} \\
\operatorname{diam}\left(K_{I}\right)= \begin{cases}r_{I} \operatorname{diam}(K) & \text { for } I \neq \emptyset \\
\operatorname{diam}(K) & \text { for } I=\emptyset\end{cases}
\end{gathered}
$$

We denote by $r_{\max }$ and $r_{\min }$ the maximum and the minimum of the contraction factors.

The similarity dimension $\alpha$ is the unique real number satisfying the equation $\sum_{i=1}^{N} r_{i}{ }^{\alpha}=$ 1.

The sequence space (or the code space) $\Omega$ is given by: $\Omega=\prod_{1}^{\infty}\{1, \cdots, N\}$. Let $C_{i_{1} \cdots i_{n}}=$ $\left(i_{1}, \ldots, i_{n}\right) \times \prod_{n+1}^{\infty}\{1, \cdots, N\}$ denote the cylinder set in $\Omega$ whose first $n$ coordinates are $\left(i_{1}, \ldots, i_{n}\right)$. We define a metric $\rho$ on $\Omega$ the following way:

$$
\rho\left(\left(i_{n}\right),\left(j_{n}\right)\right)= \begin{cases}1 & \text { if } i_{1} \neq j_{1} \\ r_{i_{1}} \cdots r_{i_{m}} & \text { if } i_{k}=j_{k} \quad 1 \leq k \leq m \text { and } i_{m+1} \neq j_{m+1} \\ 0 & \text { if } i_{k}=j_{k} \forall k\end{cases}
$$

With respect to this $\rho$ on $\Omega$ the cylinder sets are open (and closed). Since the cylinder sets generate the Borel sets of $\Omega$, we define a measure on $\Omega$ by defining it on the cylinder sets and extending it to the whole of $\Omega$. On the cylinder sets, we require that the measure $\nu$ have the property $\nu\left(C_{i_{1} \cdots i_{n}}\right)=\left(r_{i_{1}} \cdots r_{i_{n}}\right)^{\alpha}$. Again, for this metric $\rho, \nu$ is the Hausdorff $\alpha$-measure on $\Omega$ and has the property that $\mathcal{H}^{\alpha}(\Omega)=\nu(\Omega)=1$ and the Hausdorff dimension of $\Omega$ is $\operatorname{dim}_{H} \Omega=\alpha$ [3]. Corresponding to the similitudes $f_{i}$ on $K$, we define the similitudes $\overline{f_{i}}$ on $\Omega$ by $\overline{f_{i}}\left(\left(i_{1}, i_{2}, \cdots\right)\right)=\left(i, i_{1}, i_{2}, \cdots\right)$. They have the same corresponding contraction factors $r_{i}$. Let the function $g: \Omega \longrightarrow K$ map the following way: $g\left(\left(i_{1}, i_{2}, \cdots\right)\right)=\lim _{n \rightarrow \infty} f_{i_{1}} \circ f_{i_{2}} \circ \cdots \circ f_{i_{n}}(K)$. Then $g$ is a well-defined, continuous, Lipschitz map with Lipschitz constant $\operatorname{diam}(K)$. We define the image measure $\mu$ on $K$ through the map $g$. Let $\mu(A)=\nu\left(g^{-1}(A)\right)$ for $A \subset K$. The measure $\mu$ has been widely studied and seems to have its roots in the folklore of this subject. It plays a significant role in [1] and [14]. Since $g\left(C_{i}\right)=K_{i}$ but $g^{-1}\left(K_{i}\right) \supset C_{i}$, we find that $\mu\left(K_{i}\right) \geq r_{i}{ }^{\alpha}$. In fact $\mu\left(K_{i}\right)=r_{i}{ }^{\alpha}$ is equivalent to the condition $\mu\left(K_{i} \cap K_{j}\right)=0$ for all $i \neq j$. It is also known that if $\mu\left(K_{i} \cap K_{j}\right)=0$ for all $i \neq j$, then $\mu\left(K_{I} \cap K_{J}\right)=0$ for all $I, J$ incomparable. Similarly, if $\mu\left(K_{i}\right)=r_{i}{ }^{\alpha}$ for $i \in\{1, \cdots, N\}$, then $\mu\left(K_{I}\right)=r_{I}{ }^{\alpha}$ for all $I \in \mathcal{I}$.

We now define the similarity boundary of the set $K$ denoted by $\partial_{s} K$.

$$
B=\partial_{s} K=\bigcup_{j \neq k} f_{j}^{-1}\left(f_{j}(K) \cap f_{k}(K)\right)
$$

For example in the case of the Koch curve (Example 4.2 ) in $\mathbb{R}^{2}$, the boundary $\partial_{s} K$ is just the two endpoints. This is not the same as the topological boundary of the Koch curve 
which the curve itself. On the other hand, the von Koch curve is homeomorphic to an interval and the endpoints are its boundary as a manifold. So, even in this case there is some justification in the analogy of $\partial_{s} K$ being its boundary.

Since $\partial_{s} K$ is closed, the set $U$ defined as $U=K \backslash B$ is open in $K$. As we will see later $U$ can also be defined another way, independent of the definition of $\partial_{s} K$. We say that the similarity boundary $B$ is inverse invariant if $f_{i}^{-1}(B) \cap K \subseteq B$ for all $i$.

Lastly, we define the strong open set condition in $K \overline{\mathrm{SOSC}}_{K}$. If there exists a set $U$ open in $K$ such that $f_{i}(U) \subseteq U$ for all $i$ and $f_{i}(U) \cap K_{j}=\emptyset$ for all $i \neq j$, then we say that the IFS satisfies the $\overline{\mathrm{SOSC}}_{K}$. We would like to point out here that while we are weakening the SOSC by requiring only that $U$ be open in $K$, we are also requiring that the images of the open set $U$ be disjoint from the images of the set $K$ itself. But as long as we restrict ourselves to $K$, this is only a slightly stronger version of the SOSC. Moreover, when the similitudes are homeomorphisms of the ambient space as would be the case if $X$ is $\mathbb{R}^{n}$, then SOSC implies $\overline{\mathrm{SOSC}}_{K}$.

\section{§3. Main Results}

In this section, we use $B$ to refer to the similarity boundary and $U$ to refer to $K \backslash B$.

Lemma 3.1. $f_{i}^{-1}(B) \cap K \subseteq B$ if and only if $f_{i}(U) \subseteq U$.

Proof. $(\Rightarrow)$ Let $f_{i}^{-1}(B) \cap K \subseteq B$. Then,

$$
K \backslash f_{i}^{-1}(B) \supseteq K \backslash B=U
$$

That is,

$$
f_{i}(K) \backslash B \supseteq f_{i}(U)
$$

Since $U=K \backslash B, U \supset f_{i}(K) \backslash B$. So $U \supset f_{i}(U)$ by (1).

$(\Leftarrow)$ Conversely, let $f_{i}(U) \subseteq U$. That is, $f_{i}(U) \subseteq K \backslash B$.

So

$$
\begin{gathered}
K \backslash f_{i}(U) \supseteq B \\
f_{i}^{-1}\left[K \backslash f_{i}(U)\right] \cap K \supseteq f_{i}^{-1}(B) \cap K \\
{\left[f_{i}^{-1}(K) \backslash U\right] \cap K \supseteq f_{i}^{-1}(B) \cap K} \\
f_{i}^{-1}(K) \cap K \backslash U \supseteq f_{i}^{-1}(B) \cap K
\end{gathered}
$$

Since $B=K \backslash U$,

$$
B \supset f_{i}^{-1}(K) \cap K \backslash U
$$

This implies $B \supset f_{i}^{-1}(B) \cap K$. 
Lemma 3.2. $U=K \backslash B=\bigcup_{i=1}^{N} f_{i}^{-1}\left(U_{i}\right) \cap K$ where, $U_{i}=K \backslash \bigcup_{i \neq j} f_{j}(K)$.

Proof.

$$
\begin{aligned}
f_{i}^{-1}\left(U_{i}\right) \cap K & =f_{i}^{-1}\left[K \backslash \bigcup_{j \neq i} f_{j}(K)\right] \cap K \\
& =\left[f_{i}^{-1}(K) \backslash \bigcup_{j \neq i} f_{i}^{-1}\left(f_{j}(K)\right)\right] \cap K \\
& =f_{i}^{-1}(K) \cap K \backslash \bigcup_{j \neq i} f_{i}^{-1}\left(f_{j}(K)\right) \cap K \\
& =K \backslash \bigcup_{j \neq i} f_{i}^{-1}\left(f_{j}(K)\right) \cap K
\end{aligned}
$$

Now taking the union over all the $i=1, \cdots, N$,

$$
\begin{aligned}
\bigcup_{i=1}^{N} f_{i}^{-1}\left(U_{i}\right) \cap K & =K \backslash \bigcup_{i=1}^{N} \bigcup_{j \neq i} f_{i}^{-1}\left(f_{j}(K)\right) \cap K \\
& =K \backslash B
\end{aligned}
$$

This proves the Lemma.

Lemma 3.3. $f_{i}(U) \cap f_{j}(K)=\emptyset$ for all $i \neq j$

Proof.

$$
\begin{aligned}
f_{i}(U) \cap f_{j}(K) & =f_{i}(K \backslash B) \cap f_{j}(K) \\
& =\left[f_{i}(K) \backslash f_{i}(B)\right] \cap\left[f_{j}(K)\right] \\
& =\left[f_{i}(K) \cap f_{j}(K)\right] \backslash\left[f_{i}(B)\right]
\end{aligned}
$$

By the definition of $B$,

$$
\begin{aligned}
f_{i}(B) & =f_{i}\left(\bigcup_{j \neq k} f_{j}^{-1}\left(f_{j}(K) \cap f_{k}(K)\right)\right) \\
& \supseteq f_{i}(K) \cap f_{j}(K)
\end{aligned}
$$

So, $f_{i}(U) \cap f_{j}(K)=\emptyset \quad \forall i \neq j$.

Thus, with the above result, if the set $U$ is non-empty, it satisfies the $\overline{\mathrm{SOSC}}_{K}$.

Lemma 3.4. Let $\mu\left(K_{i}\right)=r_{i}{ }^{\alpha} \quad \forall i=1, \cdots, N$. Then for any $\mu$-measurable set $A \subseteq K$, $\mu\left(f_{I}(A)\right)=r_{I}^{\alpha} \mu(A)$ for any $I \in \mathcal{I}$.

Proof. Let $A \subseteq K$ be a $\mu$-measurable set. For any $I \in \mathcal{I}$, consider the following commutative diagram: 


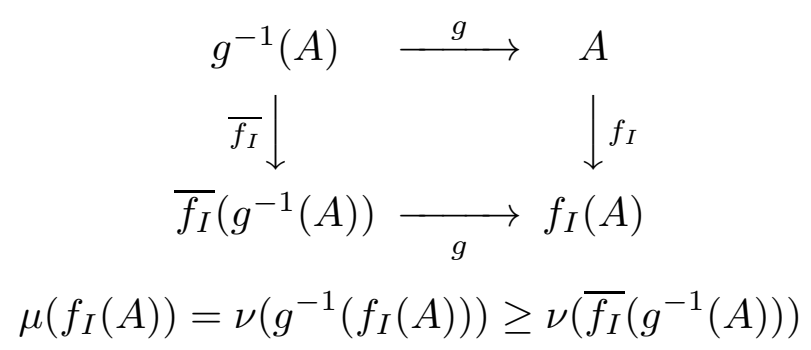

where the $\overline{f_{I}}$ are the corresponding similitudes defined on $\Omega$.

Since on $\Omega, \nu$ is the Hausdorff measure:

$$
\nu\left(\overline{f_{I}}\left(g^{-1}(A)\right)\right)=r_{I}^{\alpha} \nu\left(g^{-1}(A)\right)=r_{I}^{\alpha} \mu(A)
$$

So,

$$
\mu\left(f_{I}(A)\right) \geq r_{I}^{\alpha} \mu(A)
$$

Since $A \subseteq K$,

$$
\begin{gathered}
K_{I}=\left(K_{I} \backslash f_{I}(A)\right) \cup f_{I}(A) \\
K_{I} \backslash f_{I}(A)=f_{I}(K \backslash A)
\end{gathered}
$$

So,

$$
\begin{gathered}
K_{I}=f_{I}(K \backslash A) \cup f_{I}(A) \\
\mu\left(K_{I}\right)=\mu\left(f_{I}(K \backslash A)\right)+\mu\left(f_{I}(A)\right)
\end{gathered}
$$

since the union is disjoint.

That is,

$$
r_{I}^{\alpha}=\mu\left(f_{I}(K \backslash A)\right)+\mu\left(f_{I}(A)\right)
$$

By (1):

$$
r_{I}^{\alpha} \geq r_{I}^{\alpha} \mu(K \backslash A)+r_{I}^{\alpha} \mu(A)=r_{I}^{\alpha} \mu((K \backslash A) \cup A)=r_{I}{ }^{\alpha}
$$

Hence,

$$
\mu\left(f_{I}(A)\right)=r_{I}^{\alpha} \mu(A)
$$

which proves the Lemma.

We are now ready to prove the main result.

Theorem 3.5. Let $\left(f_{i}^{-1}(B) \cap K\right) \subseteq B \forall i$. That is, let the similarity boundary be inverse invariant. Then the following are equivalent:

(1) $\overline{S O S C}_{K}$ is satisfied by $\left\{f_{i}\right\}_{i=1}^{N}$

(2) $\operatorname{dim}_{H} K=\alpha$

(3) $U \neq \emptyset$

(4) $\operatorname{int} B=\emptyset$

(5) $\mu\left(K_{i}\right)=r_{i}{ }^{\alpha} \quad i=1, \cdots, N$

(6) $\mu\left(K_{i} \cap K_{j}\right)=0$

(7) $\mu(B)=0$ 
Proof.

$(1) \Longrightarrow(2)$ :

The following proof is a slight modification of the proof to be found in [15]. The hypotheses have been changed by doing away with the condition that the similitudes be onto and also that the SOSC be satisfied. Here, we assume only that the $\overline{\mathrm{SOSC}}_{K}$ is satisfied and the collection of similitudes is not assumed to be bijective. Let $U$ be open in $K$ such that $U$ satisfies all the rquirements of the $\overline{\mathrm{SOSC}}_{K}$. Also let $\beta<\alpha$ be the Hausdorff dimension of $K$. Let $I \in \mathcal{I}$ be such that $K_{I} \subseteq U$. So for every integer $n \geq 0$, the sets $\left\{K_{J I}\right\}_{|J|=n}$ are pairwise disjoint. Hence the self-similar set corresponding to the IFS $\left\{f_{J I}\right\}_{|J|=n}$ has disjoint images under the similitudes which generate it. If the similarity dimension of this self-similar set is assumed to be $\alpha_{n}$, then its Hausdorff dimension will also be the same. Thus,

$$
\alpha_{n} \leq \operatorname{dim}_{H} K \leq \alpha
$$

Since $\alpha_{n}$ is the similarity dimension

$$
1=\sum_{|J|=n} r_{J I}^{\alpha_{n}}=r_{I}^{\alpha_{n}} \sum_{|J|=n} r_{J}^{\alpha_{n}}
$$

So we get

$$
\begin{aligned}
r_{I}^{-\alpha_{n}} & =\sum_{|J|=n} r_{J}^{\alpha_{n}} \\
& \geq \sum_{|J|=n} r_{J}^{\beta} \\
& =\sum_{|J|=n} r_{J}^{\alpha} r_{J}^{\beta-\alpha} \\
& \geq \sum_{|J|=n} r_{J}^{\alpha} r_{\max }^{n(\beta-\alpha)} \\
& =r_{\max } n(\beta-\alpha)
\end{aligned}
$$

But on the one hand, while $r_{\max }{ }^{n(\beta-\alpha)}$ goes to infinity as $\mathrm{n}$ goes to infinity, on the other $r_{I}^{-\alpha_{n}}$ is bounded by $r_{I}^{-\beta}$. This contradiction implies that the Hausdorff dimension of $K$ is $\alpha$.

$(2) \Longrightarrow(3)$ :

If $U=\emptyset$, then $K=B$ and so, int $B \neq \emptyset$. Then for $x \in B$, there exists $x \in G \subset B$ open in $K$.

$$
B=\bigcup_{i \neq j} f_{i}^{-1}\left(f_{j}(K)\right) \cap K
$$

By the Baire Category Theorem, there exists $O \subseteq G$ open, such that,

$$
O \subseteq f_{i}^{-1}\left(f_{j}(K)\right) \cap K
$$

Since $g^{-1}(O) \subseteq \Omega$, is open in $\Omega$, and the cylinder sets form a basis for the Borel sets in $\Omega$, there exists an $I \in \mathcal{I}$ such that for some cylinder set $C_{I}$,

$$
C_{I} \subseteq g^{-1}(O) \subseteq g^{-1}\left(f_{i}^{-1}\left(f_{j}(K)\right) \cap K\right)
$$




$$
\Longrightarrow g\left(C_{I}\right)=K_{I} \subseteq f_{i}^{-1}\left(f_{j}(K)\right) \cap K
$$

That is,

$$
f_{i}\left(K_{I}\right) \subseteq f_{j}(K) \cap f_{i}(K)
$$

So $K_{i I} \subseteq K_{j} \quad$ where clearly $i I \notin j$. This means that the Hausdorff dimension $\operatorname{dim}_{H} K<$ $\alpha$ which is a contradiction.

$(3) \Longrightarrow(4)$ :

Let $U \neq \emptyset$ and $\operatorname{int} B \neq \emptyset$. We have proved, in $(2) \Longrightarrow(3)$ that $\operatorname{int} B \neq \emptyset$ implies that $\operatorname{dim}_{H} K<\alpha$. On the other hand, $\left(f_{i}^{-1}(B) \cap K\right) \subseteq B \Longrightarrow f_{i}(U) \subseteq U$ where, by assumption, $U \neq \emptyset$. So $U$ satisfies the $\overline{\operatorname{SOSC}}_{K}$. This implies, from $(1) \Longrightarrow(2)$ that $\operatorname{dim}_{H} K=\alpha$, which is a contradiction. So $\operatorname{int} B=\emptyset$.

$(4) \Longrightarrow(1)$ :

Let $\operatorname{int} B=\emptyset$. If $U=\emptyset$, then $K=B$ and that is contrary to the assumption. So $U \neq \emptyset$. So $U$ satisfies $\overline{\operatorname{SOSC}}_{K}$.

$(1) \Longrightarrow(5)$ :

Claim. $g\left(\overline{f_{i}}(A)\right)=f_{i}(g(A))$ for all $A \subseteq \Omega$.

Let $\left(i_{1}, i_{2}, \cdots\right)=x \in A$. Then,

$$
\begin{aligned}
\overline{f_{i}}(x) & =\left(i, i_{1}, i_{2}, \cdots\right) \\
g\left(\overline{f_{i}}(x)\right. & =\lim _{n \rightarrow \infty} f_{i} \circ f_{i_{1}} \circ \cdots \circ f_{i_{n}}(K) \\
& =f_{i}\left(\lim _{n \rightarrow \infty} f_{i_{1}} \circ \cdots \circ f_{i_{n}}(K)\right) \\
& =f_{i}(g(x))
\end{aligned}
$$

Since $x \in A$ is arbitarary, $g \circ \overline{f_{i}}=f_{i} \circ g$.

Now assume that $U \neq \emptyset$, that $f_{i}(U) \subseteq U$ for all $i$ and that $f_{i}(U) \cap f_{j}(U)=\emptyset$ for all $i \neq j$. Recall that $U=K \backslash B$ with $B=\partial_{s} K$. Thus, $U$ is open in $K$. Then, $\bigcup_{i=1}^{N} f_{i}(U) \subseteq U \subseteq K$.

Consider,

$$
\begin{aligned}
\sigma^{-1}\left(g^{-1}(U)\right) & =\bigcup_{i=1}^{N} \overline{f_{i}}\left(g^{-1}(U)\right) \\
g\left(\sigma^{-1}\left(g^{-1}(U)\right)\right) & =g\left(\bigcup_{i=1}^{N} \overline{f_{i}}\left(g^{-1}(U)\right)\right) \\
& =\bigcup_{i=1}^{N} f_{i}(U) \text { by Claim } \\
& \subseteq U
\end{aligned}
$$


This implies $\sigma^{-1}\left(g^{-1}(U)\right) \subseteq g^{-1}(U)$. So $g^{-1}(U)$ is invariant under the inverse of the Bernoulli shift map.

Therefore, by the Ergodic Theorem, $\nu\left(g^{-1}(U)\right)=1$. That is, $\mu(U)=1$. But, since $\nu$ is the Hausdorff measure on $\Omega$ and $\overline{f_{i}}$ is a similitude,

$$
\nu\left(\overline{f_{i}}\left(g^{-1}(U)\right)\right)=r_{i}{ }^{\alpha} \nu\left(g^{-1}(U)\right)=r_{i}{ }^{\alpha}
$$

By the Claim,

$$
g\left(\overline{f_{i}}\left(g^{-1}(U)\right)\right)=f_{i}(U)
$$

So,

$$
\overline{f_{i}}\left(g^{-1}(U)\right) \subseteq g^{-1}\left(f_{i}(U)\right)
$$

From (2),

$$
\mu\left(f_{i}(U)\right)=\nu\left(g^{-1}\left(f_{i}(U)\right)\right) \geq \nu\left(\overline{f_{i}}\left(g^{-1}(U)\right)\right)=r_{i}{ }^{\alpha}
$$

Since $\left\{f_{i}(U)\right\}_{i=1}^{N}$ is a disjoint collection,

$$
1=\mu(U) \geq \mu\left(\bigcup_{i=1}^{N} f_{i}(U)\right)=\sum_{i=1}^{N} \mu\left(f_{i}(U)\right) \geq \sum_{i=1}^{N} r_{i}{ }^{\alpha}=1
$$

This forces $\mu\left(f_{i}(U)\right)=r_{i}{ }^{\alpha}$ and $\mu(U)=\mu\left(\bigcup_{i=1}^{N} f_{i}(U)\right)$.

Now consider,

$$
\mu\left(f_{1}(K)\right)+\mu\left(f_{2}(K)\right)+\cdots+\mu\left(f_{N}(K)\right) \geq 1
$$

But $f_{1}(K) \cap f_{j}(U)=\emptyset$ for all $j \neq 1$. So we have,

$$
\begin{aligned}
1=\mu(K) & \geq \mu\left(f_{1}(K) \bigcup\left(\bigcup_{i=2}^{N} f_{i}(U)\right)\right) \\
& =\mu\left(f_{1}(K)\right)+\sum_{i=2}^{N} \mu\left(f_{i}(U)\right) \geq 1
\end{aligned}
$$

This forces $\mu\left(f_{1}(K)\right)=r_{1}{ }^{\alpha}$. The same argument can be repeatedly used to show that $\mu\left(f_{i}(K)\right)=r_{i}^{\alpha} \quad \forall i$.

$(5) \Longrightarrow(6)$ :

Let $\mu\left(K_{i}\right)=r_{i}^{\alpha} \quad i=1, \cdots, N$.

$$
\nu\left(g^{-1}\left(K_{i}\right) \backslash C_{i}\right)=\nu\left(g^{-1}\left(K_{i}\right)\right)-\nu\left(C_{i}\right)=0
$$

$g^{-1}\left(K_{i}\right)$ may be written in the following way,

$$
g^{-1}\left(K_{i}\right)=\left(g^{-1}\left(K_{i}\right) \backslash C_{i}\right) \cup C_{i}
$$


It follows that for any $i \neq j$,

$$
\nu\left(g^{-1}\left(K_{i}\right) \cap C_{j}\right)=\nu\left(\left(g^{-1}\left(K_{i}\right) \backslash C_{i}\right) \cap C_{j}\right) \cup \nu\left(C_{i} \cap C_{j}\right)=0
$$

So,

$$
\begin{aligned}
\mu\left(K_{i} \cap K_{j}\right) & =\nu\left(g^{-1}\left(K_{i}\right) \cap g^{-1}\left(K_{j}\right)\right) \\
& =\nu\left(\left(g^{-1}\left(K_{i}\right) \backslash C_{i}\right) \cap g^{-1}\left(K_{j}\right)\right)+\nu\left(C_{i} \cap g^{-1}\left(K_{j}\right)\right)=0
\end{aligned}
$$

$(6) \Longrightarrow(5)$ :

Let $\mu\left(K_{i} \cap K_{j}\right)=0$. That is, $\nu\left(g^{-1}\left(K_{i}\right) \cap g^{-1}\left(K_{j}\right)\right)=0$. Since $\mu\left(K_{i}\right)=\nu\left(g^{-1}\left(K_{i}\right)\right) \geq r_{i}^{\alpha}$, we need to show that $\mu\left(K_{i}\right) \leq r_{i}{ }^{\alpha}$.

Consider,

$$
\begin{aligned}
g^{-1}\left(K_{i}\right) & =C_{i} \bigcup\left[\bigcup_{j \neq i} g^{-1}\left(K_{i}\right) \cap C_{j}\right] \\
& \subseteq C_{i} \bigcup\left[\bigcup_{j \neq i} g^{-1}\left(K_{i}\right) \cap g^{-1}\left(K_{j}\right)\right]
\end{aligned}
$$

By hypothesis $\nu\left(\bigcup_{j \neq i} g^{-1}\left(K_{i}\right) \cap g^{-1}\left(K_{j}\right)\right)=0$. So, $\mu\left(K_{i}\right)=\nu\left(g^{-1}\left(K_{i}\right)\right) \leq r_{i}{ }^{\alpha}$.

$(6) \Longrightarrow(7)$ :

Let $\mu\left(K_{i} \cap K_{j}\right)=0$.

$$
B=\bigcap_{i \neq j} f_{i}^{-1}\left(K_{i} \cap K_{j}\right)
$$

Let $f_{i}^{-1}\left(K_{i} \cap K_{j}\right) \cap K=A$. Then $K_{i} \cap K_{j}=f_{i}(A)$.

In order to prove that $\mu(B)=0$, we need only prove that $\mu(A)=0$. Now $\mu\left(f_{i}(A)\right)=0$ by hypothesis. But $0=\mu\left(f_{i}(A)\right)=r_{i}{ }^{\alpha} \mu(A)$ by Lemma 3.4. So $\mu(A)=0$ and hence $\mu(B)=0$.

$(3) \Longrightarrow(7)$ :

$U \neq \emptyset$ and $f_{i}(U) \subseteq U$ implies, by the Ergodic Theorem, that $\mu(U)=1$. Therefore $\mu(B)=\mu(K \backslash U)=0$.

$(7) \Longrightarrow(3)$ :

$\mu(B)=0$ implies that $\operatorname{int} B=\emptyset$. But if $U=\emptyset$, then $K=B$ which is a contradiction. So $U \neq \emptyset$.

Remark. The proof of (1) implies (5), that is, the $\overline{\mathrm{SOSC}}_{K}$ implying that for all $i \neq j$ $\mu\left(K_{i} \cap K_{j}\right)=0$ did not use the invariance of the similarity boundary and as such stands by itself. This is also true of the proof of the equivalence of (5) and (6). It is possible to prove 
Theorem 3.5 by proving fewer implications than above. We have included more proofs than necessary for the insights they provide.

We now prove that in the case of self-similar tiles in Euclidean space, when the similarity boundary is inverse invariant, it is the same as the topological boundary. Intuitively, this is what we would expect and also what would be a desirable property in the similarity boundary.

Theorem 3.6. Let $K$ be a self-similar tile in $n$-dimensional Euclidean space $\mathbb{R}^{n}$ with similarity dimension $n$. If the similarity boundary of $K$ is inverse invariant, then

$$
\partial_{s} K=\partial K
$$

where $\partial K$ represents the topological boundary.

Proof.

We first prove that $\partial_{s} K \subseteq \partial K$. Since $K$ is a tile in $\mathbb{R}^{n}$, it has non-empty interior and since the similarity dimension of $K$ is $n$, the Hausdorff dimension is also $n$. Let int $K=O$.

Claim. $\operatorname{int}\left(f_{i}(K)\right) \cap \operatorname{int}\left(f_{j}(K)\right)=\emptyset$ for all $i \neq j$.

Proof of Claim. Since the similitudes are open maps in Euclidean space, $\operatorname{int}\left(f_{i}(K)\right)=$ $O_{i}$. So we need to show that $O_{i} \cap O_{j}=\emptyset$ for all $i \neq j$. Let us assume the contrary. Then, since $O_{i} \cap O_{j}$ is an open set, $\lambda\left(O_{i} \cap O_{j}\right)>0$, where $\lambda$ is the Lebesgue measure in $\mathbb{R}^{n}$. But, from [1]:

$$
0=\lambda\left(K_{i} \cap K_{j}\right) \geq \lambda\left(O_{i} \cap O_{j}\right)>0
$$

which is a contradiction that proves the Claim.

$O_{i} \cap O_{j}=\emptyset$ implies that

$$
K_{i} \cap K_{j}=\partial K_{i} \cap \partial K_{j}
$$

But, since $f_{i}$ is a homeomorphism on $\mathbb{R}^{n}, f_{i}^{-1}\left(\partial K_{i}\right)=\partial K$. This means that $\partial K_{i}=f_{i}(\partial K)$. So from (1):

$$
K_{i} \cap K_{j}=f_{i}(\partial K) \cap f_{j}(\partial K)
$$

Now, in order to prove that $\partial_{s} K \subseteq \partial K$, we need to prove that for every $i \neq j, f_{i}^{-1}\left(K_{i} \cap\right.$ $\left.K_{j}\right) \subseteq \partial K$. That is, we need to prove that for every $i \neq j, K_{i} \cap K_{j} \subseteq f_{i}(\partial K)$ which follows from (2). So $\partial_{s} K \subseteq \partial K$.

We now prove the containment in the other direction. That is, $\partial K \subseteq \partial_{s} K$. $\partial_{s} K \subseteq \partial K$ is a closed set. Let $x \in \partial K \backslash \partial_{s} K$.

Claim. Let $x \in \partial K \backslash \partial_{s} K$. For all $i, f_{i}(x) \in \partial K$.

Proof of Claim. If $f_{i}(x) \in \operatorname{int} K$, then, since $f_{i}(x) \in \partial K_{i}, f_{i}(x) \in f_{j}(K)$ for some $j \neq i$. By the definition of the similarity boundary this would mean that $x \in \partial_{s} K$ and that is a contradiction. So for all $i, f_{i}(x) \in \partial K$. This proves the Claim. 
But on the other hand, if $f_{i}(x) \in \partial_{s} K$, then $x \in f_{i}^{-1}\left(\partial_{s} K\right) \subseteq \partial_{s} K$ by the assumption on the similarity boundary. But this contradicts the choice of $x$. Since $x \in \partial K \backslash \partial_{s} K$ was arbitarary, this proves that $f_{i}\left(\partial K \backslash \partial_{s} K\right) \subseteq \partial K \backslash \partial_{s} K$ for all $i$. So $f_{i}\left(\overline{\partial K \backslash \partial_{s} K}\right) \subseteq \overline{\partial K \backslash \partial_{s} K}$ for all $i$. Let $A=\overline{\partial K \backslash \partial_{s} K}$. So $\overline{\partial K \backslash \partial_{s} K}$ is forward invariant. That is, $\bigcup_{i=1}^{N} f_{i}(A) \subseteq A$. This implies that $K \subseteq A$ which is a contradiction by the Theorem 3.5 above. So $\partial K=\partial_{s} K$.

\section{$\S 4$. CONCLUSION}

It is worthwhile to point out that in Theorem 3.6 above, $\partial_{s} K \subseteq \partial K$ is true without the similarity boundary being necessarily inverse invariant. However, the inverse containment is not always true if the similarity boundary is not inverse invariant. We illustrate this point with the following example.

Example 4.1. In $\mathbb{R}^{2}$, we define the following four similitudes: $f_{1}$ is contraction towards $(0,1)$ by a factor $1 / 2, f_{2}$ is contraction towards $(1,1)$ by $1 / 2, f_{3}$ is contraction towards $(0,0)$ by $1 / 2$ and $f_{4}$ is contraction towards $(1,0)$ by $1 / 2$. Then the self-similar set we get is the unit square in $\mathbb{R}^{2}$. It can be shown that its similarity boundary is the outer edges of the square and that it is inverse invariant. It also coincides with the topological boundary. Now, we modify the similitudes slightly the following way: we add a rotation by -90 degrees to $f_{2}$, a rotation by 90 degrees to $f_{3}$ and a rotation by 180 degrees to $f_{4}$. We still get the same invariant set. But now, the similarity boundary is just the line segments connecting $(1,1)$ to $(1,0)$ and $(0,0)$ to $(1,0)$. Also, the similarity boundary is no longer inverse invariant and it no longer coincides with the topological boundary of the unit square.

As a further point of interest, it has been proved that the Hausdorff dimension of $\partial_{s} K$ is in fact strictly less than the similarity dimension $\alpha$ of the set $K$ [9]. We would like to mention that the complement of the similarity boundary in $K$ is not necessarily the maximal open set (open in $K$ ) satisfying the requirements of the $\overline{\mathrm{SOSC}}_{K}$. As an illustration, consider the following example.

Example 4.2. The von Koch curve is the invariant set of the IFS consisting of the following contraction similitudes $f_{1}, f_{2}, f_{3}$ and $f_{4}$ defined the following way. Let $f_{1}$ be contraction towards the origin $(0,0)$ by a factor $1 / 3$. Let $f_{2}$ be contraction by a factor $1 / 3$ towards the origin, rotation by 60 degrees followed by translation to the right by $1 / 3$ units so that $(0,0)$ goes to $(1 / 3,0)$. Let $f_{3}$ be contraction by a factor $1 / 3$ towards the origin, rotation by -60 degrees followed by a translation taking $(0,0)$ to $\left(\frac{1}{2}, \frac{\sqrt{3}}{6}\right)$. Let $f_{4}$ be contraction towards the point $(1,0)$ by a factor $1 / 3$. Let $U$ be the set formed by deleting the origin from the curve. Then $U$ is open in $K$. It can be easily verified that it contains the complement of the similarity boundary. Furthermore, it satisfies the requirements of the $\overline{\mathrm{SOSC}}_{K}$.

Lastly, we take another look at an example in [15] of a self-similar set $K$ in Hilbert space which has the property that it satisfies the SOSC but has zero Hausdorff $\alpha$ measure. The example that follows is basically the one in [15]. However, in our analysis, there is no need for the similitudes to be onto and so the definitions are somewhat simpler. 
Example 4.3. We consider the $l_{1}$ space endowed with the corresponding norm.

$$
l_{1}=\left\{\left(x_{n}\right): x_{n} \in \mathbb{R}, \sum_{n=1}^{\infty}\left|x_{n}\right|<\infty\right\}
$$

Let $\left\{e_{n}\right\}_{n=1}^{\infty}$ denote the unit vectors which form a basis for the space. We define the similitudes $f_{1}, f_{2}$ and $f_{3}$ the following way for $x=\left(x_{1}, x_{2}, x_{3}, \cdots\right) \in l_{1}$,

$$
\begin{gathered}
f_{1}\left(x_{1}, x_{2}, x_{3}, \cdots\right)=\left(0, x_{1} / 2,0, x_{2} / 2,0, x_{3} / 2,0, \cdots\right) \\
f_{2}\left(x_{1}, x_{2}, x_{3}, \cdots\right)=\left(0,0, x_{1} / 2,0, x_{2} / 2,0, x_{3} / 2,0, \cdots\right) \\
f_{3}\left(x_{1}, x_{2}, x_{3}, \cdots\right)=\left(\left(x_{1}+1\right) / 2, x_{2} / 2, x_{3} / 2, \cdots\right)
\end{gathered}
$$

$f_{1}, f_{2}$ and $f_{3}$ are contracting similitudes on $l_{1}$ with contraction factors $r_{1}=r_{2}=r_{3}=1 / 2$. $f_{1}$ and $f_{2}$ have as their fixed point the origin and since $f_{3}$ can be written as $f_{3}(x)=$ $e_{1}+1 / 2\left(x-e_{1}\right)$, the fixed point of $f_{3}$ is $e_{1}$. It is clear from the definition of $f_{1}$ and $f_{2}$ that $K_{1} \cap K_{2}=(0,0,0, \cdots)$. Also, if $f_{3}\left(x_{1}, x_{2}, x_{3}, \cdots\right)=f_{1}\left(y_{1}, y_{2}, y_{3}, \cdots\right)$, then $x_{1}=-1$. Now let $P$ be the set of points in $l_{1}$ with non-negative coordinates. Then, $f_{i}(P) \subseteq P$ for $i=1,2,3$. Since $P$ is a closed set, this implies that $K \subseteq P$ and so, $K$ cannot contain any points with negative coordinates. This in turn implies that $K_{3} \cap K_{1}=\emptyset$. The same argument also shows that $K_{3} \cap K_{2}=\emptyset$. So $f_{i}(K) \cap f_{j}(K)$ is either empty or the origin. Since the origin is the fixed point of both $f_{1}$ and $f_{2}, f_{1}^{-1}(0)=0=f_{2}^{-1}(0) . f_{3}^{-1}(0)$ is a point whose first coordinate is -1 and therefore lies outside $K$. All this proves that the similarity boundary of this set $K$ is nothing but the origin and also that it is inverse invariant. Since $\mu(B)=0$, all the equivalences from Theorem 3.5 follow. So if we restrict ourselves to $K$, then instead of having to explicitly construct an open set to satisfy the SOSC, Theorem 3.5 gives us the existence of such a set. In particular, $\operatorname{dim}_{H} K=\alpha$ where $(1 / 2)^{\alpha}+(1 / 2)^{\alpha}+(1 / 2)^{\alpha}=1$. Once again, restricting ourselves to $K$, from [15], we get that $K$ has zero Hausdorff $\alpha$ measure. Hence we get the required example.

In the above example, the contraction factors could have been any $\left\{c_{1}, c_{2}, \cdots, c_{N}\right\}$ with $0<c_{i}<1$ and the analysis would have been the same. It is somewhat easier to reach the conclusions about $K$ than the analysis found in [15].

\section{REFERENCES}

[1] C. Bandt and S. Graf, Self-similar sets 7. A characterization of self-similar fractals with positive Hausdorff measure, Proc. Amer. Math. Soc. 114 (1992), 995-1001.

[2] Michael Barnsley, Fractals Everywhere, Academic Press Professinal, 1993.

[3] G. A. Edgar, Measure, Topology, and Fractal Geometry, Springer-Verlag, 1990.

[4] G. A. Edgar, Classics on Fractals, Addison-Wesley.

[5] K. J. Falconer, The Geometry of Fractal Sets, Cambridge University Press, 1985.

[6] K. J. Falconer, Fractal Geometry Mathematical Foundations and Applications, John Wiley and Sons, 1990.

[7] Marshall Hall Jr., Combinatorial Theory, Blaisdell Pub. Co., 1967.

[8] J. E. Hutchinson, Fractals and self similarity, Indiana Univ. Math. J. 30 (1981), 713-747. 
[9] J.E. Keesling, The boundaries of self-similar tiles in $\mathbb{R}^{n}$, Topology and its Applications 94 (1999), 195-205.

[10] S.P. Lalley, Travelling salesman with self-similar itenerary, Probab. Engin. Infor. Sciences 4 (1990), 1-18.

[11] D.J. Larman, A new theory of dimension, Proc. London Math. Soc. 17 (1967), no. 3, 178-192.

[12] P. Mattila, Geometry of Sets and Measures in Euclidean Spaces Fractals and rectifiability, Cambridge University Press, 1995.

[13] P.A.P. Moran, Additive functions of intervals and Hausdorff measure, Proc. Cambridge Philos. Soc. 42 (1946), 15-23.

[14] A. Schief, Separation properties for self-similar sets, Proc. Amer. Math. Soc. 122 (1994), 111-115.

[15] A. Schief, Self-similar sets in complete metric spaces, Proc. Amer. Math. Soc. 124 (1996), 481-490.

[16] S. Stella, On Hausdorff dimension of recurrent net fractals, Proc. Amer. Math. Soc. 116 (1992), 389-400.

University of Florida, Department of Mathematics, P.O. Box 118105, 358 Little Hall, Gainesville, FL 32611-8105, USA

E-mail address: jek@math.ufl.edu

University of Florida, Department of Mathematics, P.O. Box 118105, 358 Little Hall, Gainesville, FL 32611-8105, USA

E-mail address: cak@math.ufl.edu 